\title{
Percepciones, conocimiento y enseñanza de cambio climático y riesgo de desastres en universidades hondureñas
}

\author{
Laura E. Suazo ${ }^{1 *}$ y Antonio Torres-Valle ${ }^{2}$ \\ (1) Departamento de Ambiente y Desarrollo, Universidad Zamorano, Apdo.93 Tegucigalpa, Honduras. \\ (Correo-e: Isuazo@zamorano.edu; lesuazo@gmail.com) \\ (2) Instituto Superior de Tecnología y Ciencias Aplicadas, Ministerio de Ciencia, Tecnología y Medio Ambiente, La Habana, \\ Cuba. (Correo-e: atorres@instec.cu; antoniotorresvalle@gmail.com) \\ * Autor a quien debe ser dirigida la correspondencia.
}

Recibido Jul. 13, 2020; Aceptado Sep. 8, 2020; Versión final Oct. 13, 2020, Publicado Feb. 2021

\begin{abstract}
Resumen
El objetivo de este estudio es examinar las percepciones, conocimiento y enseñanza de cambio climático y riesgo de desastres de 457 docentes de universidades hondureñas. En los últimos 20 años, Honduras ha figurado entre los países con mayor vulnerabilidad al cambio climático. La herramienta RISKPERCEP sirvió para calcular la percepción del riesgo frente a desastres naturales, vulnerabilidad climática, vulnerabilidad social y respuesta a desastres. Los resultados muestran una percepción global de 2.06, con una tendencia a sobreestimación de variables: inmediatez, catastrofismo, voluntariedad, papel de la prensa, involucración e historia pasada. Se encontró subestimación para clima organizacional, comprensión del riesgo, incertidumbre y familiaridad. El 59\% de los docentes no enseñaron los temas y perciben su conocimiento como adecuado, a pesar que el $60 \%$ reportó no haber recibido capacitación. Se concluye que los resultados revelan la importancia de fortalecer el conocimiento y la investigación aplicada de la facultad de universidades hondureñas en cambio climático y riesgo de desastres.
\end{abstract}

Palabras clave: cambio climático; educación ambiental; gestión; percepción; riesgo

\section{Perceptions, knowledge, and teaching of climate change and natural disaster risk at Honduran universities}

\begin{abstract}
The main objective of the present study was to examine perceptions, knowledge, and teaching of climate change and natural disaster risk of 457 faculty members of Honduran universities. The tool RISKPERCEP was used to calculate perception of natural disasters, climate vulnerability, social vulnerability, and disaster response. The results showed that the teachers interviewed evaluated risk with an overall perception of 2.06. They had a tendency to overestimate the following variables: imminence, catastrophism, exposure, media role, involvement, and history. They underestimated risk for organizational climate, risk comprehension, uncertainty, and familiarity. Overall, 59\% of the teachers interviewed did not teach the topics examined here. But they perceived their knowledge was adequate, even though more than $60 \%$ of the informers had not received formal training on climate change or natural disaster risk. It is concluded that the results reveal the importance of strengthening the knowledge and applied research of Honduran university professors on climate change and natural disaster risk.
\end{abstract}

Keywords: climate change; environmental education; risk perception; management

Formación Universitaria Vol. 14 № $1-2021$ 


\section{INTRODUCCIÓN}

El cambio climático se define como "cambio de clima atribuido directa o indirectamente a la actividad humana que altera la composición de la atmosfera global y que se suma a la variabilidad natural del clima observada durante períodos comparables" (IPCC, 2014). El cambio climático dejó de ser un mito científico, para volverse uno de los mayores retos de la sociedad, no solo puede provocar la ocurrencia de desastres, sino también amplificar sus impactos, como ocurre en Honduras. El planeta está en peligro, la comunidad científica considera esta era como una emergencia planetaria (IPCC, 2013). Según el Grupo Intergubernamental de Expertos sobre el Cambio Climático (IPCC), la actividad humana es responsable del calentamiento global, el cual, desde la década de 1950 no tiene antecedentes en los últimos decenios a milenios (IPCC, 2013).

La emisión continua de gases de efecto invernadero (GEI) provocará mayor calentamiento y alteraciones en el sistema climático, resultando en una probabilidad de impactos graves en las personas y ecosistemas (IPCC, 2013, IPCC, 2014). La percepción de cambio climático y del riesgo asociado dependen de las interpretaciones individuales de las personas, las cuales varían según país, geografía y cultura (Lee et al., 2015), género, estatus socioeconómico (Ibarrarán et al., 2014) y exposición a información pública y científica (Lorenzoni y Pidgeon, 2006). Poca investigación se ha hecho en Honduras, un país altamente vulnerable al cambio climático y al riesgo de desastres naturales, para conocer como la facultad universitaria se prepara y afronta la problemática. El riesgo en este contexto de estudio se refiere a la probabilidad de que una amenaza climática se convierta en un desastre. La gestión integrada de riesgo de desastres (GIRD) identifica vulnerabilidades frente a las amenazas y actúa para prevenirlas o para manejar el impacto cuando el desastre es inevitable. En Honduras, la teoría GIRD no se incluye de forma transversal para todas las universidades, de tal manera que las acciones mencionadas por Gutiérrez y Sánchez (2018): aceptar, mitigar, transferir y evitar fluyan como conocimiento general en las carreras.

En los últimos años, Centroamérica y El Caribe han experimentado eventos climáticos extremos. En 1998 el paso del huracán Mitch dejó muerte y daño a la infraestructura en Centro América y especialmente en Honduras. El cambio climático afecta a Honduras, debido a su ubicación geográfica (un corredor entre dos océanos), el nivel de desarrollo (en vías), y la poca o escasa formación de la población en prevención y respuesta a desastres. Honduras ocupó entre 1997 y 2016 junto a Haití y Birmania el primer lugar entre las repúblicas con mayor afectación por eventos climáticos extremos según el Índice de Riesgo Climático de Germanwatch (Eckstein et al., 2018). Los países en vías de desarrollo es conocido que sufran la mayor afectación por el cambio climático, debido a su vulnerabilidad social y económica (Eckstein et al., 2018), menor contacto con información cientifica (Lee et al., 2015) y menor capacidad de respuesta por su alta vulnerabilidad individual y colectiva (Ibarrarán et al., 2014).

Los esfuerzos para hacerle frente al cambio climático, se enfocan en adaptación y mitigación. La mitigación se orienta principalmente hacia la reducción de GEI o hacia la mejoría de los sumideros (IPCC, 2014). Existe consenso mundial para reducir las emisiones, aunque aún no se refleje en acciones mayores (Hamin y Gurran, 2009). La adaptación al cambio climático es el proceso de ajuste al clima mediante iniciativas que reducen la vulnerabilidad del ambiente y las personas (IPCC, 2013). La falta de información climática, estudios de impacto y de vulnerabilidad, carencias y confusiones en el conocimiento, representan las mayores barreras para lograr adaptación (Hamin, 2009; Meira-Cartea y Arto-Blanco, 2014; Taylor et al., 2014). El IPCC reconoce el papel de las universidades en la formación para la reducción de la vulnerabilidad (IPCC, 2014). La educación ambiental en el sistema universitario puede influir en la formación de profesionales involucrados en respuestas sociales, económicas y tecnológicas relacionadas con el cambio climático y el manejo de riesgo de desastres (Meira-Cartea y Arto-Blanco, 2014; Solis-Espallargas y Barreto-Tovar 2020; Xavier et al., 2016). Las universidades juegan un papel clave en cumplir la agenda 2030 de desarrollo sostenible (Olaguez et al., 2019). Ese papel exige una preparación multidisciplinaria ante la creciente demanda de nuevos profesionales con habilidades en el manejo de riesgos de desastres (Henderson et al., 2017; Xavier et al., 2016).

La vulnerabilidad educativa para analizar riesgo climático como una calificación de la ausencia de programas escolares y del eje temático transversal en el currículo educativo ( Xavier et al., 2016) es alta en Honduras. En el contexto de este estudio, también se relaciona con la ausente o escasa formación académica de la facultad en el tema, a pesar que, en varios estudios la educación es el mayor predictor de la conciencia pública sobre cambio climático y riesgo de desastres a nivel mundial (Lee et al., 2015). El riesgo por desastres climáticos en Honduras es considerado alto (Eckstein et al., 2018), no solo por la frecuencia de eventos, sino por la escaza preparación, prevención y respuesta de la población. En la mayoría de las universidades la enseñanza de la gestión integrada de riesgo de desastres (GIRD) está ausente o diseminada en algunos cursos en ciertas carreras. 
En la construcción social de la vulnerabilidad, cada individuo percibe y maneja el riesgo a nivel personal y global (Lorenzoni y Pidgeon, 2006). Los aspectos sociales, físicos, económicos influyen en el grado y tipo de vulnerabilidad que enfrentan hombres y mujeres (Soares y Murillo-Licea, 2013), independiente si sean profesores universitarios o no. La percepción de riesgo varía de acuerdo con la cultura, lugar, país, la conceptualización científica que maneje la persona e historial de exposición al riesgo (Taylor et al., 2014). De hecho, el paradigma psicométrico (Prades y González, 1999) empleado para el estudio de percepción de riesgo en esta investigación, considera en su valoración factores de índole individual, de naturaleza física del riesgo y de gestión del riesgo. Está claro que una población con patrones culturales de apego al lugar de residencia subvalora los riesgos asociados a su entorno, pues la predicción y la gestión de los fenómenos naturales pasa a ser parte de su psicología socio-ambiental. Ello se convierte en factor histórico y temporal, el primero porque representa la experiencia de la comunidad en el enfrentamiento a factores medioambientales adversos y el segundo, porque se enmarca en determinadas temporadas o épocas del año (Muñoz-Duque y Arroyave, 2017). Con la fragilidad ante el cambio climático se puede aumentar la pobreza, la cual repercute en la respuesta de las personas ante eventos de riesgo (Ibarrarán et al., 2014). Pero está claro que la formación en el manejo del riesgo puede ayudar a reducir esa vulnerabilidad (Xavier, 2016).

La creación de conocimiento se basa en las creencias epistemológicas de los individuos, esas creencias no siempre son científicamente correctas (Beck et al., 2013; Cajigal et al., 2016; Taylor, et al., 2014). En general, se percibe un conocimiento limitado de la población acerca de las causas y soluciones al cambio climático (Oltra et al., 2009), que incluye al sector de los docentes universitarios, especialmente aquellos que no tienen ninguna formación en el área ambiental o de ciencias naturales. Varios estudios confirman una auto percepción de docentes con deficiencias en conocimiento ambiental; limitada disponibilidad de herramientas de enseñanza en el tema y poca investigación (Domenech-Casal, 2014;García-Rodeja y Lima, 2012; Saidón y Claverie, 2016). También poco o confuso conocimiento de maestros y alumnos sobre el cambio climático y la gestión de riesgo de desastres (Cajigal et al., 2016; Dal, et al., 2014; Meira-Cartea y Arto-Blanco, 2014; Saidón y Claverie, 2016). Esa percepción mundial, tambien se percibió para el cuerpo docente universitario en Honduras, previo a este estudio, en parte porque no se identificaron mayores iniciativas de formación en el tema de cambio climático y de GIRD.

La forma como la sociedad se adapta al cambio climático, está determinada por: "el entendimiento de valores, creencias, percepciones y normas" (Oltra et al., 2009; Gasparetto, et al., 2018). O'Connor et al., (1999) comprobó que las percepciones de riesgo influyen en las intenciones de comportamiento de las personas. Sin embargo, en varios estudios, la mayoría de las personas reportan dificultad de relacionar el impacto del cambio climático con aspectos de su vida cotidiana (Torres et al., 2017). La percepción del cambio climático como un problema sin solución puede provocar un sentimiento de desinterés y falta de eficacia de acción al nivel personal. Algunos estudios indican que las personas reaccionan, cuando lo que sucede en el entorno amenaza sus valores y creen que sus acciones contribuyen en recuperar la situación o en detener la amenaza (Gasparetto, et al., 2018). Seroussi et al., (2019), señalan tres categorías de aprendizaje de temas socioambientales, como el cambio climático: la primera es conocimiento, la segunda es creencias y la tercera es actitud. En este sentido, las universidades pueden aportar conocimiento en la respuesta a como Honduras se adapte al cambio climático.

En Honduras se percibe que la población asume una construcción de riesgos considerada como inmunidad subjetiva, en la cual se minimiza la probabilidad de peligros cotidianos, cortando la percepción de riesgos altamente probables, tal y como lo comprobaron en Cuba, el Dr. Antonio Torres y otros en la última decada. Oltra et al., (2009) ayuda a entender esa postura con el hecho de que los impactos mayores pueden ocurrir en 30, 50 o 100 años, puede ser parte de la indiferencia en la población actual. Aunque el calentamiento global es un término común, la conciencia pública para abordar el cambio climático se considera baja (Norton y Leaman, 2004). El escepticismo sobre el cambio climático y el riesgo de desastre asociado incluye también a las universidades por la limitada acción en enseñanza, investigación e involucramiento operativo (Leal, et al., 2019). En el caso de Honduras, la inclusión del tema de cambio climático y riesgo de desastres ha sido impulsado en su mayoría por organismos de cooperación internacional para el desarrollo, y no como una acción endógena de las universidades.

EI IPCC reconoce un rol primordial de las personas y sus conocimientos en la adaptación como proceso de gestión de riesgo (IPCC, 2014). El incremento de la conciencia ambiental y de la acción pro clima y pro prevención de desastres requiere mayores iniciativas de comunicación y de educación (Leal et al., 2019; Lee et al., 2015) dirigidas hacia valores y actitudes (Parker y Muñoz, 2012). El fortalecimiento de la enseñanza ambiental a través de la inclusión curricular de cambio climático y gestión de riesgo de desastres es fundamental (Parker y Muñoz, 2012). El accionar de las universidades es fundamental para la implementación de la agenda 2030 que busca el 
desarrollo sostenible. El involucramiento de las universidades como centros de aprendizaje, investigación, innovación y vinculación, debe ir más allá de edificios eco eficientes y campus verdes, hace falta que esta temática se incluya en oferta curricular (Beck et al., 2013; Leal et al., 2019; Reid, A.,(2019). El rol de las universidades, se puede fortalecer con políticas institucionales específicas en respuesta al cambio climático (Henderson, et al., 2017). Aunque la generación de información climática en Centroamérica se percibe en aumento, en Honduras, la formación de capacidades técnicas desde las universidades se considera insuficiente. Todavía existe cierto escepticismo entre docentes sobre el tema, hay ausencia temática en la mayoría de las ofertas académicas de las universidades y la creación de redes y alianzas entre universidades, empresas y la sociedad requiere fortalecimiento y sostenibilidad.

Las 20 universidades hondureñas (seis públicas y 14 privadas) conforman el Comité Interinstitucional de Universidades en Ciencias Ambientales de Honduras (CICA) y la Red de Universidades de Latino América y El Caribe para la Reducción de Riesgo de Desastres (REDULAC-RRD) de Honduras. Existen experiencias pioneras de universidades que han iniciado acciones de revisión, adecuación y transversalización curricular en Adaptación al Cambio Climático (ACC) y GIRD, como son: Universidad Zamorano, Universidad Pedagógica Nacional Francisco Morazán, Universidad Nacional Autónoma de Honduras y Universidad Metropolitana. El apoyo de organismos de cooperación internacional como la Agencia Suiza para el Desarrollo y la Cooperación (COSUDE), ha sido clave en el fortalecimiento de la academia universitaria hondureña para iniciar acciones de inclusión de los temas. En el 2019 el CICA presentó una solicitud formal ante la Dirección de Educación Superior (DES) de Honduras, para la inclusión de cambio climático y GIRD como asignatura de pregrado universitario.

A pesar de las iniciativas, se percibe ausencia de ambas temáticas en la formación general básica y en la oferta académica en el sistema universitario de Honduras. El conocer como los docentes universitarios hondureños perciben y enseñan el cambio climático y el riesgo de desastre es clave para impulsar un plan de fortalecimiento educativo nacional en el tema. Este estudio tomó como referencia una caracterización del conocimiento y la percepción de riesgo sobre cambio climático en la red de escuelas cubanas asociadas a la UNESCO (Torres, et al., 2017). El objetivo de la investigación fue: analizar las percepciones, el conocimiento y la enseñanza de cambio climático y gestión de riesgo de desastres, de docentes en el nivel de pregrado de las universidades hondureñas.

\section{METODOLOGÍA}

El estudio contó con la participación de 457 docentes de pregrado de 12 universidades hondureñas, quienes respondieron de forma voluntaria una encuesta en línea. Al ser la percepción de riesgo, una medida subjetiva, se buscó un programa que permitiera cuantificar dicha subjetividad. Es decir que permitiera adaptar las cualidades de percepción a cantidades. Para ello se utilizó la herramienta informática RISKPERCEP versión 2.0, facilitado por el Dr. Antonio Torres de la Universidad de La Habana. Las variables analizadas en el estudio de Torres et al., (2017) sirvieron de base para el presente estudio (Tabla 1).

Las variables se clasificaron en tres tipos tomando como referencia el estudio en Cuba por Torres, et al., (2017): i) las que se relacionan con el individuo (Individual), ii) las que se refieren a la naturaleza del riesgo o riesgo físico (Naturaleza) y iii) las que se relacionan con la gestión del riesgo o riesgo gestionado (Gestión) (Torres et al., 2017). A partir de ese estudio, también se conoce con anticipación sobre el comportamiento de las variables. Por ejemplo, el potencial catastrófico, el pánico generado y la inmediatez de las consecuencias se comportan de forma directamente proporcional a la percepción. Otras actúan de forma inversa como la familiaridad, la capacidad de control y la reversibilidad. La variable comprensión del riesgo muestra un comportamiento extremo, ello indica que la subestiman los expertos, como los no especialistas en el tema.

EI programa RISKPERCEP calcula un promedio de los datos en cada pregunta correspondiente a una variable específica. Para ello, utiliza valores cualitativos ordinales, a los que asigna valores numéricos de 1, 2 o 3 según haya sido la respuesta. Las preguntas de características cerradas comprendieron respuestas ordenadas unidireccionalmente en tres escalas. Esto permitió lograr una correlación con la escala de percepción de riesgo asociada de tres niveles, donde 1 significa subestimación del riesgo y 3 sobreestimación, el 2 la estimación adecuada. Lo anterior aplica a preguntas que evolucionan de forma directa a la percepción asociada. Cuando las variables evolucionan de manera diferente, el programa realiza ajustes para su consideración (Torres et al., 2017).

Las respuestas se recopilaron a través de la plataforma de Survey Monkey, para su posterior análisis usando el Programa IBM SPSS Statistics y el Programa Excel. La encuesta contó con 57 preguntas, entre estilos tipo Liker y preguntas abiertas, 41 de esas preguntas alimentaron el cálculo de percepción de riesgo. El resto de las preguntas se analizaron de forma descriptiva. El programa RISKPERCEP calcula un estimador no estadístico 
llamado Score Ponderado, que representa una tendencia colectiva hacia una opinión grupal (Torres, y otros, 2017).También se determinaron las dispersiones específicas de cada variable con respecto a su valor de Score. En el marco de la metodología de análisis, los resultados de percepción por variables son independientes entre sí. Esta aproximación es necesaria pues, aunque se reconoce esta dependencia, es imposible cuantificarla sin introducir una alta subjetividad en el estudio.

Tabla 1: Variables de percepción de riesgo asociado al cambio climático. Fuente: Adaptado de Torres, et al., (2017).

\begin{tabular}{|c|c|c|c|c|}
\hline № & Variable (CÓDIGO) & Comportamiento & Relación de la percepción de riesgo & Tipo \\
\hline 1 & $\begin{array}{l}\text { Familiaridad } \\
\text { (FAMI) }\end{array}$ & Inverso & $\begin{array}{l}\text { Mayor familiaridad-menor } \\
\text { percepción de riesgo }\end{array}$ & \\
\hline 2 & Comprensión del riesgo (COM) & Extremo & $\begin{array}{l}\text { Elevado y/o escaso conocimiento- } \\
\text { menor percepción }\end{array}$ & \\
\hline 3 & $\begin{array}{l}\text { Incertidumbre } \\
\text { (INCE) }\end{array}$ & Directo & $\begin{array}{l}\text { Mayor incertidumbre-Mayor } \\
\text { percepción }\end{array}$ & \\
\hline 4 & $\begin{array}{l}\text { Involucración Personal } \\
\text { (INVO) }\end{array}$ & Directo & $\begin{array}{l}\text { Mayor involucración-mayor } \\
\text { percepción }\end{array}$ & \\
\hline 5 & Controlabilidad (CONT) & Inverso & $\begin{array}{l}\text { Mayor controlabilidad-menor } \\
\text { percepción }\end{array}$ & \\
\hline 6 & Voluntariedad (VOLU) & Inverso & $\begin{array}{l}\text { Mayor voluntariedad-menor } \\
\text { percepción }\end{array}$ & Individual \\
\hline 7 & Potencial catastrófico (CATA) & Directo & Mayor potencial-mayor percepción & \multirow{5}{*}{ Naturaleza } \\
\hline 8 & $\begin{array}{l}\text { Inmediatez de las consecuencias } \\
\text { (INME) }\end{array}$ & Directo & $\begin{array}{l}\text { Mayor inmediatez-mayor } \\
\text { percepción }\end{array}$ & \\
\hline 9 & $\begin{array}{l}\text { Historia pasada de accidentes } \\
\text { (HIST) }\end{array}$ & Directo & $\begin{array}{l}\text { Mas historia pasada-mayor } \\
\text { percepción }\end{array}$ & \\
\hline 10 & Pánico (PANI) & Directo & Más pánico-mayor percepción & \\
\hline 11 & $\begin{array}{l}\text { Reversibilidad de las } \\
\text { consecuencias (REVE) }\end{array}$ & Inverso & $\begin{array}{l}\text { Mayor reversibilidad menor } \\
\text { percepción }\end{array}$ & \\
\hline 12 & $\begin{array}{l}\text { Confianza en las Instituciones } \\
\text { (INST) }\end{array}$ & Directo & Mayor confianza-mayor percepción & \multirow{5}{*}{ Gestión } \\
\hline 13 & Beneficios (BENE) & Inverso & $\begin{array}{l}\text { Mayores beneficios-Menor } \\
\text { percepción }\end{array}$ & \\
\hline 14 & Inequidad Riesgo-Beneficio (RI-B) & Directo & $\begin{array}{l}\text { Mayor Inequidad Riesgo-Beneficio- } \\
\text { Mayor Percepción }\end{array}$ & \\
\hline 15 & Papel de la Prensa (PREN) & Directo & $\begin{array}{l}\text { Mejor rol de los medios de difusión- } \\
\text { Mayor percepción del riesgo }\end{array}$ & \\
\hline 16 & Clima organizacional (CLIM) & Directo & $\begin{array}{l}\text { Mejor clima organizacional respecto } \\
\text { a la seguridad-mayor percepción }\end{array}$ & \\
\hline
\end{tabular}

\section{RESULTADOS}

El total de encuestados fue de 457 informantes. El 52\% de los informantes (238) fueron hombres y el $48 \%$ (219) mujeres. Los informantes provinieron de doce universidades, $59 \%$ de universidades públicas y el $41 \%$ de universidades privadas. La edad promedio de los informantes fue de 41 años, con una edad mínima de 24 años y una edad máxima de 67 años. Los informantes contaron con 9.34 años de enseñanza en promedio, una moda de 2 años, un mínimo de 1 año, un máximo de 33 años y una Desviación Típica de 7.55 años. El 67\% reportaron tener entre 10 o menos años de experiencia docente. El 53\% de los encuestados contó con el grado de maestría, seguido por un título de pregrado, ya sea ingeniería o licenciatura (39\%). Solamente un $6 \%$ contó con un grado de doctorado. La figura 1 muestra la distribución de la formación académica de los informantes. 


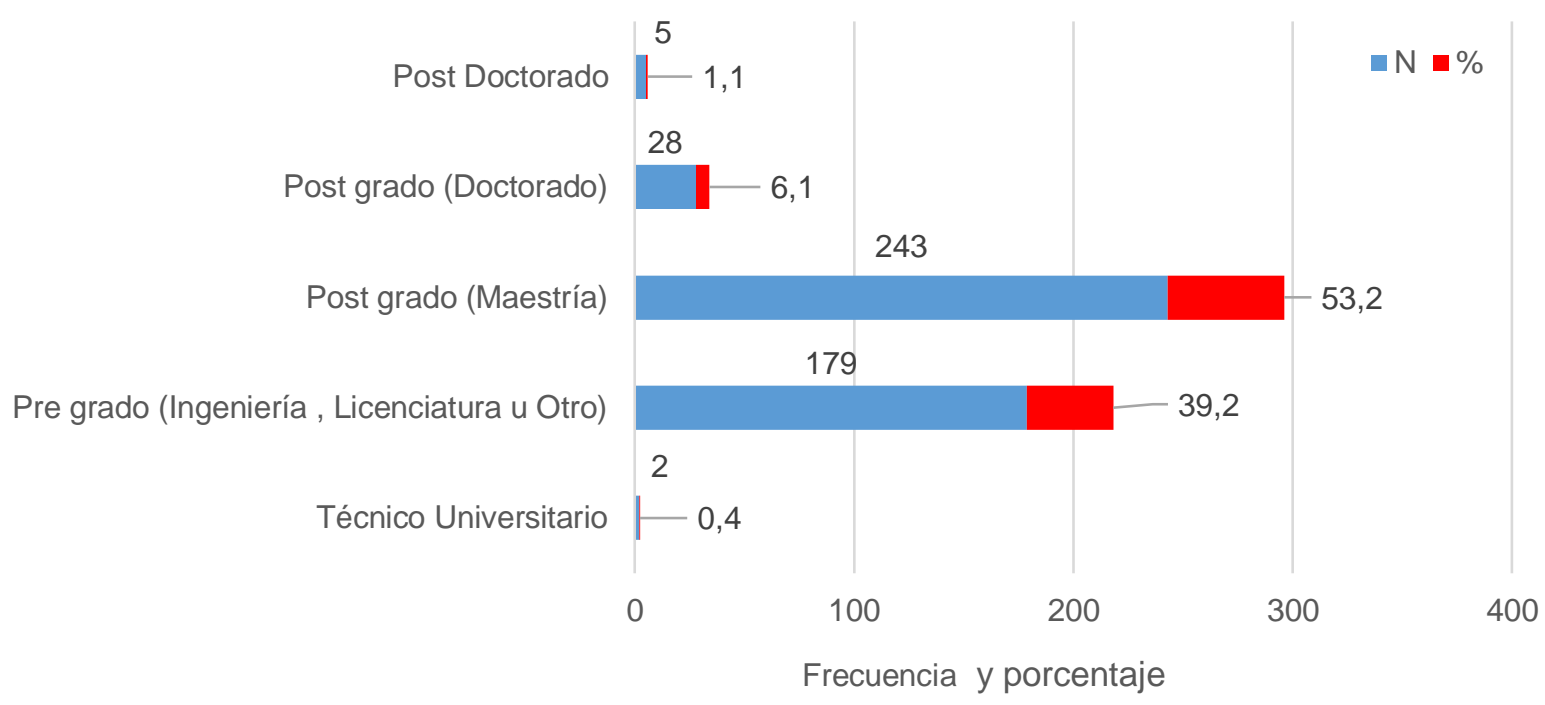

Fig. 1: Formación académica de los docentes informantes

La percepción global del riesgo para el grupo estudiado fue de 2.06 cercana a la adecuada (2.0), con una tendencia a sobreestimación de variables: inmediatez, catastrofismo, voluntariedad, papel de la prensa, involucración e historia pasada. La figura 2 muestra la distribución de los perfiles de percepción de riesgo. Se encontró subestimación para las variables: clima organizacional, comprensión del riesgo, incertidumbre y familiaridad. El conocimiento sobre cambio climático se examinó por medio de las variables incertidumbre (INCE), comprensión del riesgo (COMP) y el clima organizacional (CLIM). Con un valor de (2.80), los docentes reconocen que los riesgos del cambio climático se están manifestando rápidamente. El $90 \%$ de los docentes perciben que los desastres naturales actuales relacionados con el cambio climático serán más frecuentes en las próximas décadas y el $77 \%$ consideran que los impactos se manifestarán en el corto plazo. La mayoría de los encuestados calificó como catastróficos los riesgos asociados al cambio climático (2.69). Con un puntaje de 2.44, los docentes reconocen de forma negativa el papel de los medios en brindar información científica sobre el cambio climático y sus consecuencias. La mayoría (64\%) considera que la información brindada al público es poca o insuficiente. Entre las variables de percepción que alcanzaron mayor índice de subestimación del riesgo (menor estimación) fueron: Clima organizacional (1.45), Comprensión del riesgo (1.59), Incertidumbre (1.62) y Familiaridad (1.60).

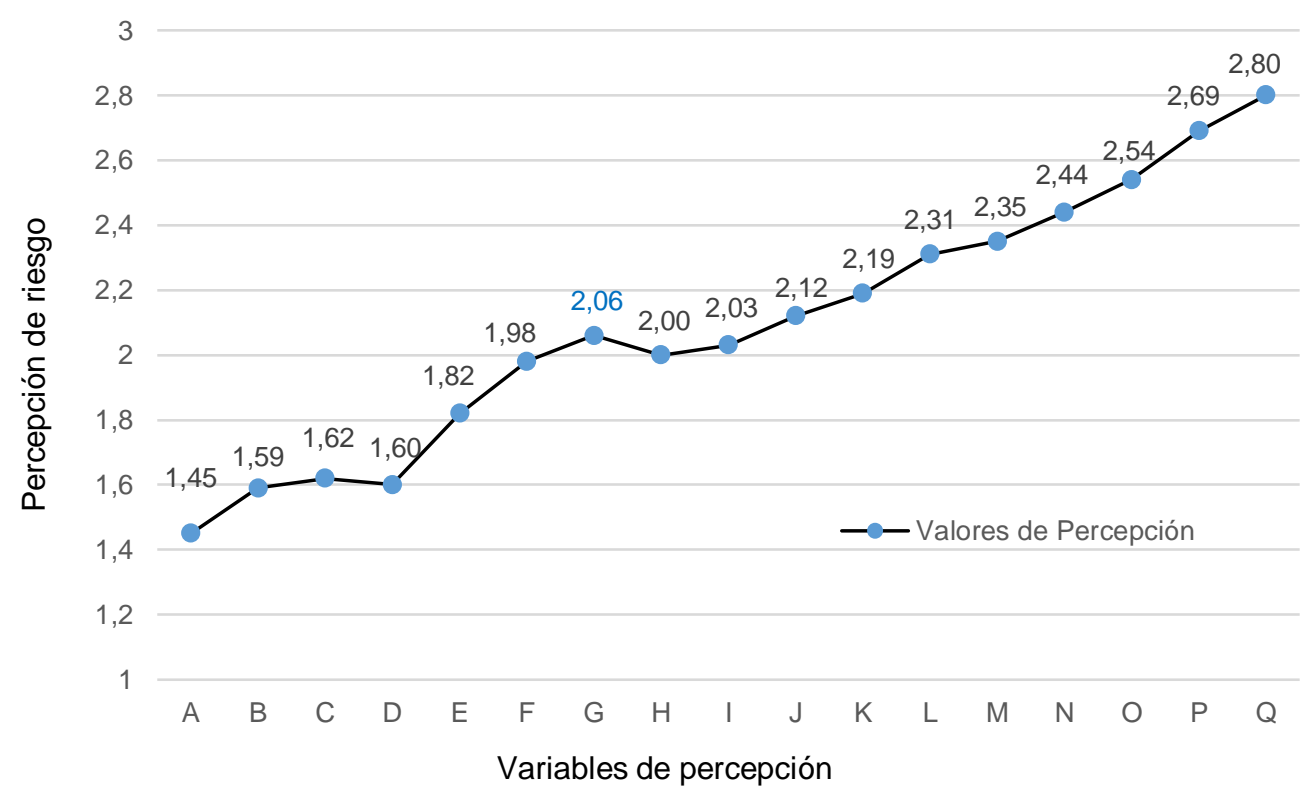

A) Clima organizacional

B) Comprensión del

riesgo

C) Incertidumbre

D) Familiaridad

E) Confianza en Instituciones

F) Beneficios

G) Score Global

H) Controlabilidad

I) Reversibilidad

J) Inequidad riesgos Beneficios

K) Pánico

L) Historia pasada

M) Involucración

N) Papel de la prensa

O) Voluntariedad

P) Catastrofismo

Q) Inmediatez

Fig. 2: Perfil de riesgos para variables de percepción 
En incertidumbre (INCE) se analizó la percepción de los docentes sobre el origen del cambio climático y fase de la ciencia en el tema. Con un valor de 1.62, la subestimación desde este punto de vista refleja una comprensión que el conocimiento de la ciencia sobre el fenómeno es claro y existe confianza en los estudios que sobre el tema se han hecho. El $87 \%$ acertó en su respuesta al considerar que el origen del cambio climático se debe a actividades humanas y al resultado de la variabilidad natural del clima. El $21 \%$ considera que la ciencia se encuentra en una fase anticipada de conocimiento sobre el tema. Hubo un $84 \%$ que consideran que las acciones para mitigar y/o adaptarse al cambio climático deben conectar con estrategias de desarrollo sostenible.

En comprensión del riesgo (COMP), la mayoría de los docentes consideran su nivel de preparación y conocimientos como bajo. De igual forma, consideran como bajo su nivel de preparación sobre metodologías y aspectos organizativos en la formación de estudiantes en el tema. El 77\% de los informantes coinciden en su respuesta sobre el concepto de desarrollo sostenible. Hubo un 19\% que interpretó el concepto desde: "se logra preservar el medio ambiente y conservar los recursos sin alteraciones" y un $4 \%$ desde: "se logra un crecimiento económico continuo, sin limitaciones en sus volúmenes o en el tiempo que satisfaga las necesidades crecientes de los seres humanos".

El $51 \%$ percibe como adecuado su nivel de preparación y conocimiento sobre el cambio climático, mientras que el $43 \%$ considera bajo. Un $54 \%$ calificó como bajo o insuficiente su preparación y conocimiento de metodologías y aspectos organizativos que deben tomarse en cuenta para el desarrollo del trabajo sobre adaptación y mitigación con estudiantes. En la variable clima organizacional (CLIM), el $62 \%$ de los informantes consideran que la preparación metodológica de los profesores para abordar temas del cambio climático, su mitigación y adaptación es baja/insuficiente (Tabla 2). En parte se atribuye al desinterés de las instituciones universitarias en el tema.

Tabla 2: Nivel de conocimiento y preparación sobre cambio climático

\begin{tabular}{|c|c|c|c|c|c|c|}
\hline \multirow{2}{*}{$\begin{array}{l}\text { Preguntas } \\
\text { Criterio }\end{array}$} & \multicolumn{2}{|c|}{$\begin{array}{l}\text { ¿A su juicio, su nivel } \\
\text { de preparación y } \\
\text { conocimientos sobre } \\
\text { temas de cambio } \\
\text { climático, su mitigación } \\
\text { y adaptación es:? }\end{array}$} & \multicolumn{2}{|c|}{$\begin{array}{l}\text { ¿En su opinión, su nivel de preparación y } \\
\text { conocimientos de metodologías y aspectos } \\
\text { organizativos que deben tenerse en cuenta } \\
\text { para el desarrollo del trabajo con } \\
\text { estudiantes sobre el cambio climático, su } \\
\text { mitigación y adaptación es:? }\end{array}$} & \multicolumn{2}{|c|}{$\begin{array}{l}\text { ¿La preparación } \\
\text { metodológica de los } \\
\text { profesores para abordar } \\
\text { temas del cambio } \\
\text { climático, su mitigación y } \\
\text { adaptación es:? }\end{array}$} \\
\hline & Frecuencia & $\%$ & Frecuencia & $\%$ & Frecuencia & $\%$ \\
\hline Bajo/insuficiente & 198 & 43 & 245 & 54 & 285 & 62 \\
\hline Adecuado & 235 & 51 & 195 & 43 & 161 & 35 \\
\hline Elevado/Abundante & 24 & 5 & 17 & 4 & 11 & 2 \\
\hline Total & 457 & 100 & 457 & 100 & 457 & 100 \\
\hline
\end{tabular}

La mitad de los informantes (50\%) reportaron haber recibido algún tipo de capacitación en cambio climático y el $47 \%$ en gestión de riesgo de desastres. La capacitación se refiere a participación en cursos cortos presenciales, cursos en línea, charlas y foros sobre el tema. El $61 \%$ de los informantes se considera actualizado sobre los impactos proyectados para el cambio climático, como ser: reducción del agua disponible, extinción de especies, reducción de la productividad de los cultivos y aparición de nuevas enfermedades. En ese tema, un 35\% reportó haber oído medianamente de los impactos y solo un $4 \%$ desconocía. Hubo un $24 \%$ que reportó conocer sobre otros ciclos de cambio climático similares al actual en la historia de la Tierra. Este último aspecto muestra congruencia con resultados de estudios similares, en donde se reporta un conocimiento limitado y confusión en las causas y consecuencias (Oltra et al., 2009). En el estudio, el 84\% considera que existe una relación entre desastres naturales ocurridos en fechas recientes (últimas décadas), como: huracanes, inundaciones y/o sequías y el cambio climático. El $41 \%$ de los docentes encuestados incluye temática de cambio climático y gestión de riesgo de desastres en los cursos que enseña y un 59\% no lo hace. En la figura 3 se muestra la distribución de cursos en los cuales se enseña la temática estudiada. 


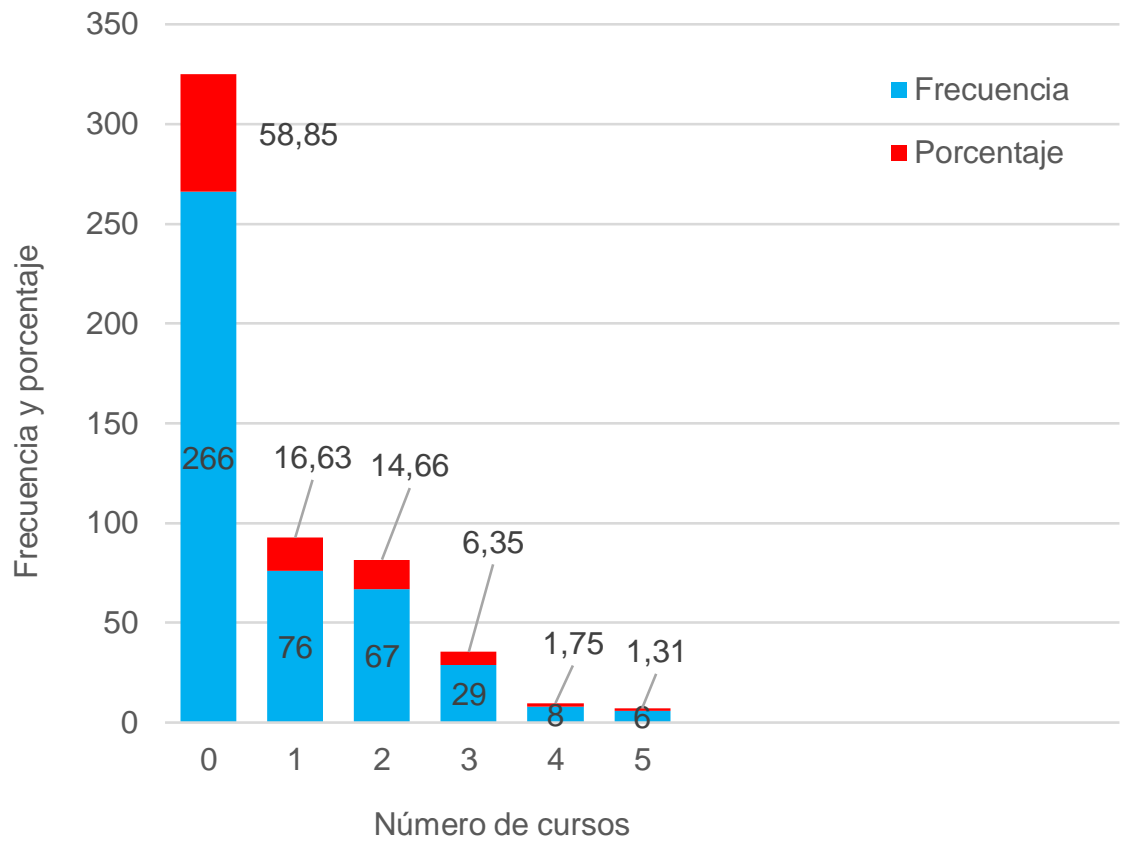

Fig. 3: Porcentaje de docentes que incluyen cambio climático y gestión de riesgo de desastres en sus cursos

En una consulta grupal se aclaró que ese abordaje es somero y se relaciona con otros temas, trabajos de investigación, presentaciones, pero en su mayoría no se refiere a clases formales con contenido específico. Al consultar sobre la presencia de los temas: desarrollo sostenible, adaptación y mitigación, cambio climático y medio ambiente en el currículo de pregrado universitario, el 59\% la consideró insuficiente. El $62 \%$ de los informantes consideró el nivel de preparación de los docentes como insuficiente. El 66\%, los docentes consideran que la preparación del estudiante es en su mayoría insuficiente. La información disponible sobre el tema para estudiantes se considera mayormente insuficiente en un $60 \%$, mientras que, el $41 \%$ de los docentes encuestados consideran como insuficiente la información disponible para los docentes. La figura 4 muestra aspectos del clima organizacional y la información y preparación de docentes y estudiantes en el tema estudiado.

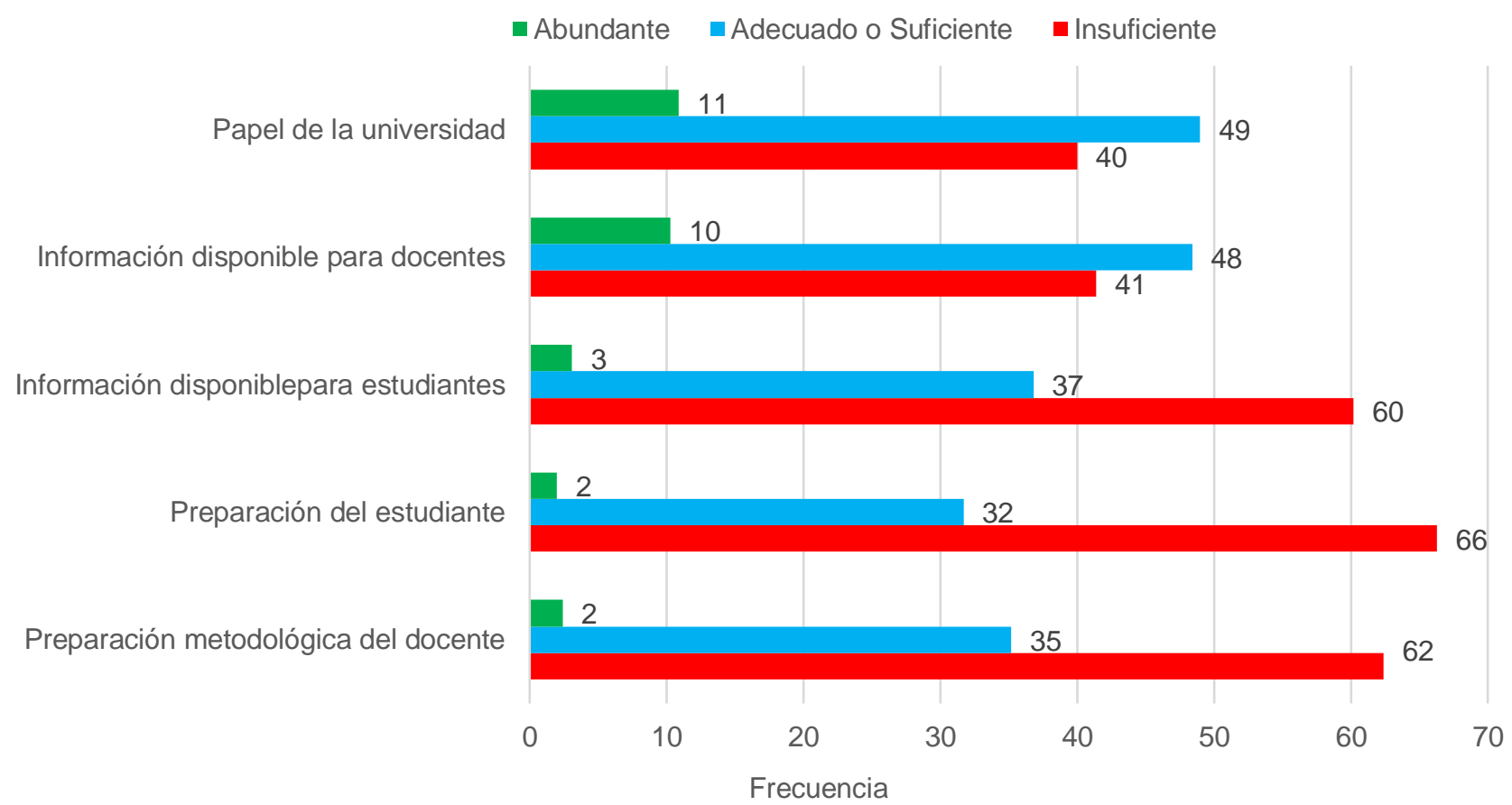

Fig. 4: Información y preparación de estudiantes y docentes sobre la temática 
En lo relacionado al desarrollo de investigaciones en temas de cambio climático, solamente el $17 \%$ ha asesorado alguna investigación, mientras que el $9 \%$ ha asesorado investigaciones en temas de cambio climático y gestión de riesgo de desastres asociado al clima. Por ser la muestra de docentes enseñando en pregrado, cabe mencionar que solo tres universidades en el estudio tienen como requisito de graduación, la realización de una investigación siguiendo el método científico: Universidad Zamorano, Universidad Nacional de Ciencias Forestales y Universidad Nacional de Agricultura. El resto de las universidades tienen otros requisitos, como reportes de prácticas sociales, monografías o proyectos especiales, en lugar de investigaciones, conocidas como tesis.

\section{DISCUSIÓN}

Los resultados encontrados en el estudio son congruentes a conclusiones generadas en el estudio cubano, que dio origen al mismo (Torres et al., 2017) y a otros estudios (Lorenzoni y Pidgeon, 2006; Parker y Muñoz, 2012; Saidon y Claverie, 2016). Los informantes del estudio cuentan, en su mayoría, con estudios avanzados de postgrado, alrededor del $50 \%$ de la muestra han recibido algún grado de capacitación en los temas estudiados. La totalidad recibe información constantemente de los medios de comunicación, aspectos que ayudan a comprender los resultados obtenidos.

La tendencia del grupo a sobreestimación del riesgo puede explicarse en parte, por la formación académica general de los docentes. También puede influir la exposición de los docentes a información sobre los temas y a actividades particulares de proyectos piloto que impulsan algunas universidades en materia de cambio climático y gestión de riesgo de desastres. Las variables con mayor índice de sobreestimación del riesgo (PGx) fueron: Inmediatez (2.80), Catastrofismo (2.69), Voluntariedad (2.54), Papel de la Prensa (2.44) e Involucración (2.35). Los resultados pueden explicarse por las siguientes razones:

Inmediatez: El valor de 2.80 para inmediatez refleja un avance en cuanto al grado de exposición a información que tienen los informantes al tema de cambio climático. De acuerdo a Lee et al., (2015), estudios de opinión pública a nivel mundial para 2007-2008 reflejaron que los países desarrollados como: Estados Unidos, Europa y Japón mostraron arriba del $90 \%$ de consciencia sobre el cambio climático, contrario a países en vías de desarrollo (Egipto, Bangladesh, Nigeria e India) en donde arriba del 65\% no estuvieron conscientes. La inmediatez percibida ha cambiado en la última década, producto de los eventos climáticos ocurridos, la experiencia personal y el historial de exposición al riesgo (Taylor et al., 2016), de los informantes en eventos de desastres climáticos.

Catastrofismo: La calificación como catastrófica de eventos asociados al cambio climático por el grupo puede deberse en parte a la experiencia previa con huracán Mitch, el cual fue de dimensiones elevadas y altamente catastrófico, con muertes y desaparecidos en Centroamérica. Se suma la ocurrencia de fenómenos climatológicos frecuentes, como tormentas tropicales, las cuales causan severos daños y muertes por la débil infraestructura, prevención y gestión del riesgo de desastre.

Voluntariedad: La voluntariedad refleja el grado de decisión del docente, de si se expone o no al riesgo, el cual es considerado como impuesto y ajeno a su voluntad. En este caso, los docentes no tienen decisión de exponerse o no al riesgo por cambio climático. Ellos consideran que tanto ellos como su familia se sienten afectados directamente por el cambio climático y por enfermedades atribuidas al cambio climático. El hecho de que Honduras ha figurado por 20 años junto a Haití y Birmania como los países con más alta vulnerabilidad al cambio climático en el mundo (Eckstein et al., 2018), puede explicar la sobreestimación de esta variable.

Papel de la prensa: La prensa juega un papel clave en la información que manejan los docentes sobre el cambio climático. En un estudio en Veracruz se encontró que el $43 \%$ de docentes entrevistados conocía muy poco sobre el cambio climático, mientras que el $93 \%$ reconoció a los medios de comunicación como la principal fuente de conocimiento. La información mediática cada vez tiene más impacto en la población (Domenech-Casal, 2014). La información mediática brinda un conocimiento básico, pero no consigue una comprensión más profunda (Saidón y Claverie, 2016), necesaria en el aprendizaje crítico y transformador. La vinculación errónea entre el deterioro de la capa de ozono y el cambio climático, así como la idea de una falta de consenso científico se considera ligada a la información de la prensa (Meira-Cartea y Arto-Blanco, 2014).

Involucración: Esta variable (2.35), tiene que ver con el grado en que el docente o su familia se sienten expuestos y/o afectados por el cambio climático. La incertidumbre ante desastres naturales y la poca preparación en prevención de riesgos, así como la exposición a situaciones como enfermedades, olas de calor, etc. influyen en la sobre estimación. También el historial de exposición a eventos climáticos y desastres influye en la sobreestimación. 
Clima organizacional: Los docentes subestiman el riesgo (1.45), reconociendo la deficiente gestión sobre los riesgos de desastres asociados al cambio climático, que ejecutan los actores e instituciones involucrados. En este caso, el sector universitario. La presencia de temas relacionados con cambio climático (adaptación y mitigación), riesgo de desastres y desarrollo sostenible fue considerada como insuficiente. Lo mismo, la preparación metodológica de docentes, la información disponible para estudiantes y para docentes, como el papel de la universidad en general en la realización de trabajos relacionados con la temática. Las políticas públicas, en este caso de educación superior, pueden ayudar a reducir la vulnerabilidad del riesgo de desastres de la población (Ibarrarán, et al., 2014, Henderson et al., 2017). El sistema universitario hondureño, no se está preparando multidisciplinariamente ante la creciente demanda de nuevos profesionales con habilidades en el manejo de riesgos de desastres (Henderson et al., 2017; Xavier et al., 2016).

Incertidumbre: Los docentes entienden que científicamente se considera que el origen del cambio climático, se debe tanto a actividades humanas, como a la variabilidad natural del clima. El hecho de que el impacto principal ocurra 30 o 100 años después, puede ser también causa de la incertidumbre (Oltra et al., 2009)

Comprensión del riesgo: El valor de 1.59, refleja bajo conocimiento en cuanto a los temas contenidos en las preguntas relacionados con el cambio climático. En su estudio global (Lee et al., 2015) confirman que educación fue un factor común entre los países, especialmente en Estados Unidos y China, para predecir conciencia sobre cambio climático. Varios estudios en diferentes países confirman este resultado, a través de una auto percepción de docentes con conocimiento deficiente, limitada disponibilidad de herramientas de enseñanza y poca o ninguna investigación (Cajigal et al., 2016; Dal, et al., 2014; Domenech-Casal, 2014; García-Rodeja y Lima, 2012; MeiraCartea y Arto-Blanco, 2014; Saidón y Claverie, 2016).

Familiaridad: El valor de 1.60 indica una subestimación del riesgo por familiaridad. En este caso, la familiaridad en parte se explica en la percepción de la mayoría (46\%) de residir a veces en zonas frecuentemente afectadas por desastres, mientras que un $29 \%$ nunca reside en dichas zonas. Los hondureños vinculan inundaciones deslizamientos por lluvia y sequía con desastres, cuya frecuencia se ha intensificado en los últimos años. El 70\% de los docentes sienten familiaridad al escuchar noticias sobre cambio climático y sus consecuencias de forma semanal.

La carencia en la formación formal o informal en los temas de cambio climático y gestión de riesgo de los docentes, se reflejará en la presencia o ausencia de cursos específicos y de contenidos en las aulas (Beck, et al., 2013). Varios docentes enseñan y hacen prácticas sobre la base de su intuición o de lo que aprenden desde los medios de comunicación (Saidón y Claverie, 2016). Varias universidades ofrecen el curso de educación ambiental o ciencias ambientales como curso electivo y en varios casos es un curso en línea. En los cursos de educación ambiental o de ciencias ambientales, puede o no incluirse la temática de cambio climático. Los alumnos no adquieren conocimiento, ni herramientas para encontrar soluciones a problemas derivados del cambio climático, como corresponde desde un modelo constructivista (Saidón y Claverie, 2016). Esto impide que los estudiantes se expongan a Estrategias de las Ciencias Básicas en la Indagación (ECBI), como lo propone (Domenech-Casal 2014) y que provocarían mayor interés, aprendizaje significativo e involucramiento. Tampoco se promueve una enseñanza en la que el conocimiento influye en las creencias y esta a su vez en las acciones pro clima (Seroussi et al., 2019).

\section{CONCLUSIONES}

De los resultados obtenidos, se derivan las siguientes conclusiones: 1) Más de la mitad de docentes perciben su conocimiento como adecuado, no enseñaron sobre los temas en los cursos que imparten, y no han recibido capacitación formal. En Honduras no existe un programa formal de actualización docente sobre los temas, por lo que los docentes buscan los medios para auto informarse. Se encontró muy poca participación docente en investigación y un percibido adecuado a bajo conocimiento de la facultad en la temática; 2) Los docentes califican el riesgo en términos cercanos a "adecuados", con una percepción global de 2.06; con sobreestimación de las variables: inmediatez, catastrofismo, voluntariedad, papel de la prensa, involucración e historia pasada y subestimación de variables como: clima organizacional, comprensión del riesgo, incertidumbre y familiaridad. Esto en parte se debe al bajo conocimiento científico sobre cambio climático, poca o ninguna formación, desconocimiento de metodologías y de aspectos organizativos desde las universidades; 3) Existe la necesidad de fortalecer el conocimiento sobre cambio climático y riesgo de desastres en el nivel de pregrado universitario, de tal manera que se refleje en la enseñanza en el aula, como en investigaciones y publicaciones relacionadas, además de iniciativas de prevención de riesgo de desastres y 4) El sistema universitario hondureño requiere fortalecimiento del conocimiento de docentes y de la oferta de carreras y programas que contribuya con mayor 
preponderancia en la reducción de daños, mitigación del riesgo de desastres y reducción de la vulnerabilidad asociada con el cambio climático. Un plan de formación para docentes, que permita replantear los programas de estudios y carreras y ampliarlos para que incluyan estas dos áreas de conocimiento de urgente enseñanza, investigación y vinculación ayudará a fortalecer el sistema universitario hondureño en materia de cambio climático y gestión de riesgo de desastres.

\section{REFERENCIAS}

Beck, A., Sinatra, G. M. y Lombardi, D., Leveraging higher-education instructors in the climate literacy effort: factors related to university faculty's propensity to teach climate change, International Journal of Climate Change: Impacts and Responses, ISSN: 1835-7156, 4(4),1-17 (2012)

Bellami, R. y Hulme, M., Beyond the tipping perception of abrut climate change and their implications, https://doi.org/10.1175/2011WCAS1081.1, Weather, Climate and Society, 3(1), 48-60 (2011)

Cajigal, E., Maldonado, A. y González, E., Construcción de conocimiento y creencias epistemológicas sobre cambio climático en docentes de nivel primaria. De la vulnerabilidad a la resiliencia, Revista Interamericana de Educación de Adultos, ISSN: 0188-8838, 38(2), 52-76 (2016)

Dal, B., Ozturk, N. y otros cuatro autores, Perception of climate change: reasons, consequenses, and willigness to act. How aware are they?, https://doi:10.20533/ijcdse.2042.6364.2014.0268, International Journal for Cross-Disciplinary Subjects in Education, 4(2), 1930-1937 (2014)

Domenech-Casal, J., Contextos de indagación y controversias socio-científicas para la enseñanza del cambio climático, Enseñanzas de las Ciencias de la Tierra, ISSN: 1132-9157, 22(3), 287-296 (2014)

Eckstein, D., Künzel, V. y Schäfer, L., Global climate risk index 2018, Germanwatch e.V. Bonn, Alemania (2019)

García-Rodeja, I. y Lima, G., Sobre el cambio climático y el cambio de los modelos de pensamiento de los alumnos, Enseñanza de las Ciencias, ISSN: 0212-4521, 30(3), 195-218 (2012)

Gasparetto, M.I., Teixeira, D. y Roazzi, A., Knowledge and beliefs about climate change and the role of the Amazonian forest among university and high school students, https://doi:10.1089/eco.2017.0050, Ecopsychology, 10(2), 106-116 (2018)

Gutiérrez, Y.E., y Sánchez- Ortiz, A., Diseño de un modelo de gestión de riesgos basado en ISO 31.000:2012 para los procesos de docencia de pregrado en una universidad chilena, https://doi:10.4067/S0718-50062018000400015, Formación universitaria, 11(4), 15-32 (2018)

Hamin, E. M. y Gurran, N., Urban form and climate change: balancing adaptation and mitigation in the U.S. and Australia, https:doi:10.1016/j.habitatint.2008.10.005, Habitat International, 33, 238-245 (2009)

Henderson, J., Bieler, A. y McKenzie, M., Climate change and the Canadian higher education system: an institutional policy analysis, Canadian Journal of Higher Education, ISSN: 0316-1218, 47(1), 1-26 (2017)

Ibarrarán, M. E., Reyes, M. y Altamirano, A., Adaptación al cambio climático como elemento de combate a la pobreza, Región y Sociedad, ISSN: 1870-3925, 26(61), 5-50 (2014)

IPCC, Stocker,T. F., Qin, D. y otros 8 Edits., Resumen para responsables de políticas, Cambio Climático 2013: Bases físicas. Contribución del grupo de trabajo I al quinto informe de evaluación del Grupo Intergubernamental de Expertos sobre el Cambio Climático, Cambridge University Press, Reino Unido y Estados Unidos (2013)

IPCC, Pachauri,R.K. y Meyer, L.A. Eds., Cambio Climático 2014: informe de síntesis. Contribución de los grupos de trabajo I, II y III al quinto informe de evaluación del Grupo Intergubernamental de Expertos sobre el Cambio Cimático, IPCC, Ginebra, Suiza (2014)

Leal, W.F., Mifsud, M. y otros cuatro autores, Climate change scepticism at universities: a global study, https://doi:10.3390/su11102981, Sustainability, 11(2981), 1-13 (2019)

Lee, T. M., Markowitz, E. M. y otros cuatro autores, Predictors of public climate change awareness and risk perceptions around the world, https://doi:10.1038/NCLIMATE2728, Nature Climate Change, 5,1014-1020 (2015)

Lorenzoni, I. y Pidgeon, N. F., Public views on climate change: European and USA perspectives, https://doi:10.1007/s10584006-9072-z, Climatic Change, 77, 73-95, (2006)

Meira-Cartea, P. A. y Arto-Blanco, M., Representaciones del cambio climático en estudiantes universitarios en España: aportes para la educación y la comunicación, https://doi:10.1590/0104-4060.38041, Educar em Revista, 3, 15-33 (2014)

Muñoz-Duque, L. A. y Arroyave, O., Percepción del riesgo y apego al lugar en población expuesta a inundación: un estudio comparativo, https://doi:10.11144/Javerianacali.PPSI15-2.pral, Pensamiento Psicológico, 15(2), 79-92, (2017)

O'Connor, R.E., Bord, R. J. y Fisher, A., Risk perceptions, general environmental beliefs, and willingness to address climate change, https://doi:10.1023/A:1007004813446, Risk Analysis, 19(3), 461-471 (1999) 
Olaguez, E., y Otros tres autores, Plan de acción a partir de la percepción en estudiantes de la universidad politécnica de Sinaloa ante el reciclaje de residuos sólidos y la educación ambiental, http://dx.doi.org/10.4067/S0718-50062019000300003, Formación Universitaria, 12(3), 3-14, (2019)

Oltra, C., Solá, R., y Otros tres autores, Cambio climático: percepciones y discursos públicos, Prisma Social- Revista de Ciencias Sociales, 2, 1-23 (2009)

Parker, C. y Muñoz, J., Elites universitarias y cambio climático, https://doi:10.1590/S1414-753X2012000200011, Ambiente y Sociedade, XV(2), 195-218 (2012)

Prades, A. y Gonzále,z, F., La percepción social del riesgo: algo más que discrepancia Expertos-Público, ISSN 0864-084X, Nucleus (26), 1-12 (1999)

Reid, A., Climate change education and research: possibilities and potentials versus problems and perils?, https://doi.org/10.1080/13504622.2019.1664075, Environmental Education Research, 25(6), 767-790 (2019)

Saidón, M. y Claverie, J., Percepciones de docentes y directores sobre los factores que promueven u obstaculizan la educación ambiental en escuelas del área metropolitana de Buenos Aires, Argentina, https://doi:10.1590/1516-731320160040010, Ciência \& Educação, 22(4), 993-1012 (2016)

Seroussi, D.E., Rothschild, N. y otros tres autores, Teachers' knowledge, beliefs and attitudes about climate change, https:// doi:10.5539/ies.v12n8p33, International Education Studies, 12(8), 33-45 (2019)

Soares, D. y Murillo-Licea, D., Gestión de riesgo de desastres, género y cambio climático. Percepciones sociales en Yucatán, México. Cuadernos de Desarrollo Rural, ISSN:0122-1450, 10(72), 181-199 (2013)

Solís-Espallargas, C. y Barreto-Tovar, C.H., La Visión de la educación ambiental de estudiantes de maestría en pedagogía en el marco de la catédra de la paz en Colombia, https://dx.doi.org/10.4067/S0718-50062020000200153, Formación universitaria, 13(2), 153-166 (2020)

Taylor, A.L., Dessai, S. y Bruine de Bruin, W., Public perception of climate risk and adaptation in the UK: a review of literature, https://doi.org/10.1016/j.crm.2014.09.001, Climate Risk Management, 4(5), 1-16 (2014)

Torres, A., Garea, B. y otros cuatro autores, Estudio de percepción de riesgo asociado al cambio climático en el sector educacional. Revista Cubana de Salud y Trabajo, 18(1), 3-13 (2017)

Xavier, M., Camolesi, T. y otros cuatro autores, Adaptation to climate change and integration of disaster risk management in business education: a case study in fundação Getulio Vargas, Brazil, https://doi:10.17230/ad-minister.28.5, AD-minister, 28, $91-126(2016)$ 